\title{
The Effect Of Interface Gradient Distribution On Unrealistic Flow In 3D Droplet Simulations
}

\author{
Anıl Yılmaz*1, Gökhan Kayansalcik ${ }^{1}$, Özgür Ertunç ${ }^{1}$ \\ ${ }^{1}$ Mechanical Engineering Department, Ozyegin University, Istanbul, Turkey \\ *Corresponding author email: anil.yilmaz.16297@ozu.edu.tr
}

\begin{abstract}
The purpose of this study is to investigate the origin of the parasitic current to provide accurate prediction of droplet surface interactions in Volume of Fluid (VOF) framework. The deformation of the droplet due to parasitic current has been the most important problem in 3D simulations. Parasitic current is influenced by curvature and surface normal estimation in the Continuum Surface Force (CSF) model. It has been shown that the number of neighboring cells of the central cell influences the gradient calculations regarding the generation of parasitic current. It has been observed that the polyhedral cell structure delivers a smoother interface gradient distribution than the cartesian cell structure. To examine the dynamics in different physical conditions, we compared simulations with base experiments to understand whether those models work. We then simulated droplet cases on stationary and moving wall conditions, and simulation results were consistent with experimental results.
\end{abstract}

\section{Keywords}

Multiphase Flow, Drop Dynamics, VOF, Parasitic Current, Gradient Calculations.

\section{Introduction}

There are many studies in the literature regarding the dynamics of droplets upon impact on different physical surfaces $[1,2]$. By adding parameters such as surface coating characteristics, surface velocities and slope, the behavior of droplets is examined experimentally and numerically [3]. Inspite of the fact that 2D and 3D simulations can be found in the literature [3, 4, 5], it is still a challenge to obtain droplet simulations that compare well with the experimental data. Difficulties of the problem include for example the time scales in the order of microseconds and small errors can be relatively large because of the small physical size of the droplets. In some droplet simulations, liquid-gas interface error arise while calculating the surface tension force with Continuum Surface Force method (CSF).

Unphysical motion of the droplet surface, which is mostly seen in 3D simulations and referred to as "parasitic current or spurious current" [6]. In 2D simulations, this problem does not demostrate itself when the droplet is examined under an axisymmetrical condition. While modeling situations where droplets impacts moving or inclined walls, simulations should be made in 3D because the axisymmetry of the flow is broken. Parasitic currents are generated when using implementations of the Continuum Surface Force (CSF) technique of [7] to model surface tension forces in Eulerian-based multi-phase simulations [6]. Thereby droplets spreading and deposition forms are disrupted. AMR (Adaptive Mesh Refinement) methods [14] based on hexahedral cells are used in multiphase solvers such as Gerris [8] and its new version Basilisk [9], which is an effective application to provide interface sharpness and reduce computational cost. Still, parasitic current cannot be improved with grid refinement or decreased computational time step [6]. Afkhami [10] used the Height Function implementation to reduce the parasitic current. Interface representation has made some accurate development with the use of Height Function and Level-Set\&VOF hybrid models [11, 12] . Recently, Eisenschmidt's model [12] are limited the primary effects of parasitic current with FS3D solver. 
Despite advances in accurate prediction of $\kappa$ (curvature) and $\vec{n}$ (surface normal) estimation with height function approximations or hybrid Level-Set\&VOF models [10,11, 12], some amount of error remains. Also, both $\kappa$ (curvature) and $\vec{n}$ (surface normal) depends on the gradient approximation of liquid fraction at the droplet surface in the CSF model. As the main source of the problem, we predict that gradient computation errors accumulate in certain directions (angles) forced by unit cell type in grid distribution. With this grid resolution and time step independent situation, encouraged us to study gradient calculation in the CSF formulation to the used computational cell.

\section{Numerical Method \\ Governing Equations}

In this study, VOF method was used for multiphase flow simulations. The continuity, momentum equations and the transport equation for the volume fraction of liquid phase are simultaneously solved. The flow is assumed to be incompressible, immiscible and newtonian. All conservation equations can be written in terms of a general transport equation by integral form :

$$
\underbrace{\frac{d}{d t} \int_{V} \rho \phi d V}_{\text {Transient Term }}+\underbrace{\int_{A} \rho \vec{U} \phi \cdot d a}_{\text {Convective Flux }}=\underbrace{\int_{A} \Gamma \nabla \phi d a}_{\text {Diffusive Flux }}+\underbrace{\int_{V} S_{\phi} d V}_{\text {Source Term }}
$$

where $\phi$ can be any scalar. It is 1 for the continuity equation and it is equal to any component of velocity vector for the momentum eqution. $V$ is the control volume and $a$ is surface area of the control volume. $\Gamma$ is diffusion coefficent in diffusive flux term and in the present problem only the diffusion of momentum is considered, i.e. it becomes the dynamic viscosity in the momentum equation.

Dynamic viscosity and mixture density equations can be written as function of volume fractions $\alpha$ forms below:

$$
\begin{aligned}
& \rho=\alpha \rho_{l}+(1-\alpha) \rho_{g} \\
& \mu=\alpha \mu_{l}+(1-\alpha) \mu_{g}
\end{aligned}
$$

Surface tension force $\left(F_{s}\right)$ in CSF model, which is one of the source terms in the momentum equation formulated by Eq. 1 . needs the calculation of (surface normal) $\vec{n}$ and (surface curvature) $\kappa$ :

$$
\begin{aligned}
& F_{s}=\sigma \kappa \vec{n} \\
& \vec{n}=(\nabla \alpha) \\
& \kappa=\nabla \cdot\left(\frac{\nabla \alpha}{|\nabla \alpha|}\right)
\end{aligned}
$$




\section{Unphysical Flows And Mesh Condition Unphysical Flows}

Equation (4) is used to calculate the surface tension forces. In the Continuum Surface Force (CSF) formulation, surface normal ( $\vec{n})$ (See Eq. 5) and curvature $(\kappa)$ (See Eq. 6 ) values are used to calculate the gradient of the volume fraction $(\alpha)$. In Fig. 1, the gradient of the volume fraction for normal impact simulations on wax surface simulation with $1 \mathrm{~mm}$ droplet diameter and 25.48 normal Weber number value is shown for cartesian and polyhedral mesh types in the propagation plane. In those simulation, there is no interface compression and the grid resolution is comparable. Unrealistic flows shows different results in simulations with polyhedral vs cartesian mesh structure. For example, volume fraction $(\alpha)$ gradient values gives smoother distribution in polyhedral mesh structure than cartesian mesh structure. Since the incorrect calculation of gradient values in the propagation plane affects the surface tension forces according to CSF equation, it disrupts the intertia-surface tension balance and causes the generation of parasitic currents. Here, polyhedral grid structure gives more accurate droplet shapes than cartesian grid structure because it provides better scalar and vector field distribution. These results confirm the hypothesis that the mesh structure in the propagation plane affects the parasitic current accumulation and formation.

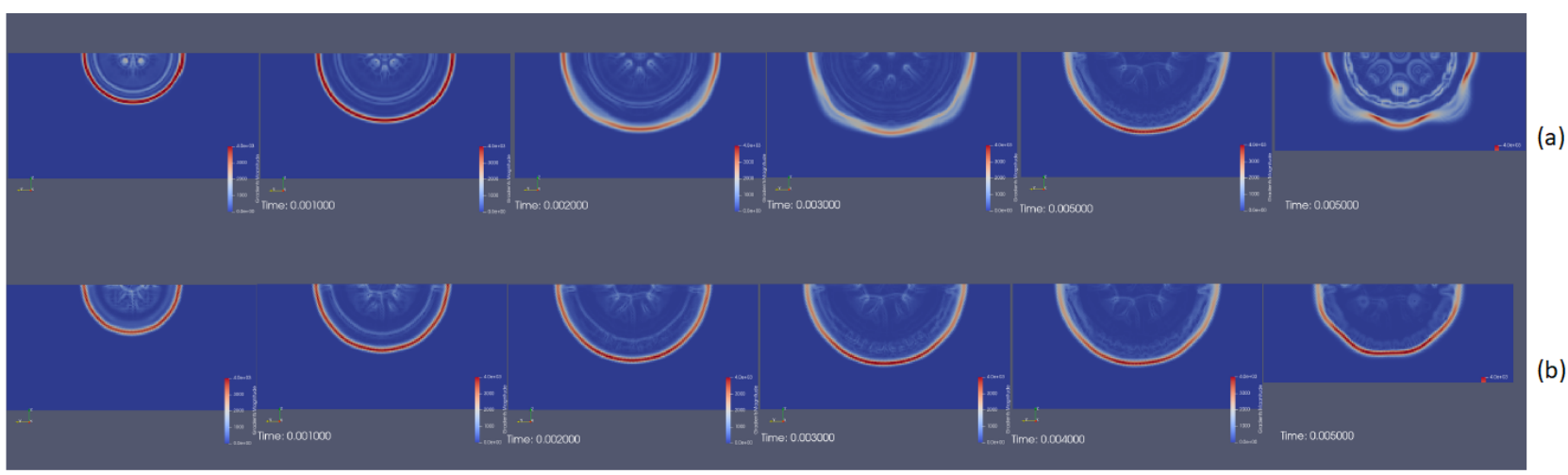

Figure 1. Time evolution of alpha gradient value at the interface on $1 \mathrm{~mm}$ droplet simulations. (a) Cartesian grid based simulation domain. (b) Polyhedral grid based simulation domain.

For the reasons mentioned above, the accumulation of gradient values and the accumulation direction of errors were analyzed numerically. The interface gradient values of the 2-dimensional and static droplets in polyhedral (hexagonal) and cartesian mesh structure were calculated. Cell-centered Gauss-Green method is used in gradient calculations. Gradient at the centroid of an element $C$ with volume $V$ is computed as:

$$
\nabla \phi_{C}=\frac{1}{V_{C}} \sum_{f \sim n b(C)} \phi_{f} S_{f}
$$

where $S$ refers to surface vector and $f$ refers to a face. $\phi$ can be any scalar as volume fraction $(\alpha)$, which is defined with avarage values according the distance ratio of the two cell sharing the face.

$$
\phi_{f}=g_{C} \phi_{C}+\left(1-g_{C}\right) \phi_{F}
$$

where $g_{C}$ is a geometric weighting factor.

It can be seen in the FIG. 2 that the gradient values calculation are affected by the type of mesh in the spreading plane. Each center has 4 neighbors and edges in the cartesian grid and 6 neighbors and edges in the polyhedral (hexagonal) grid. Hence, according to the gradient calculation formulation, polyhedral grid utilizes information from higher number of neighboring 

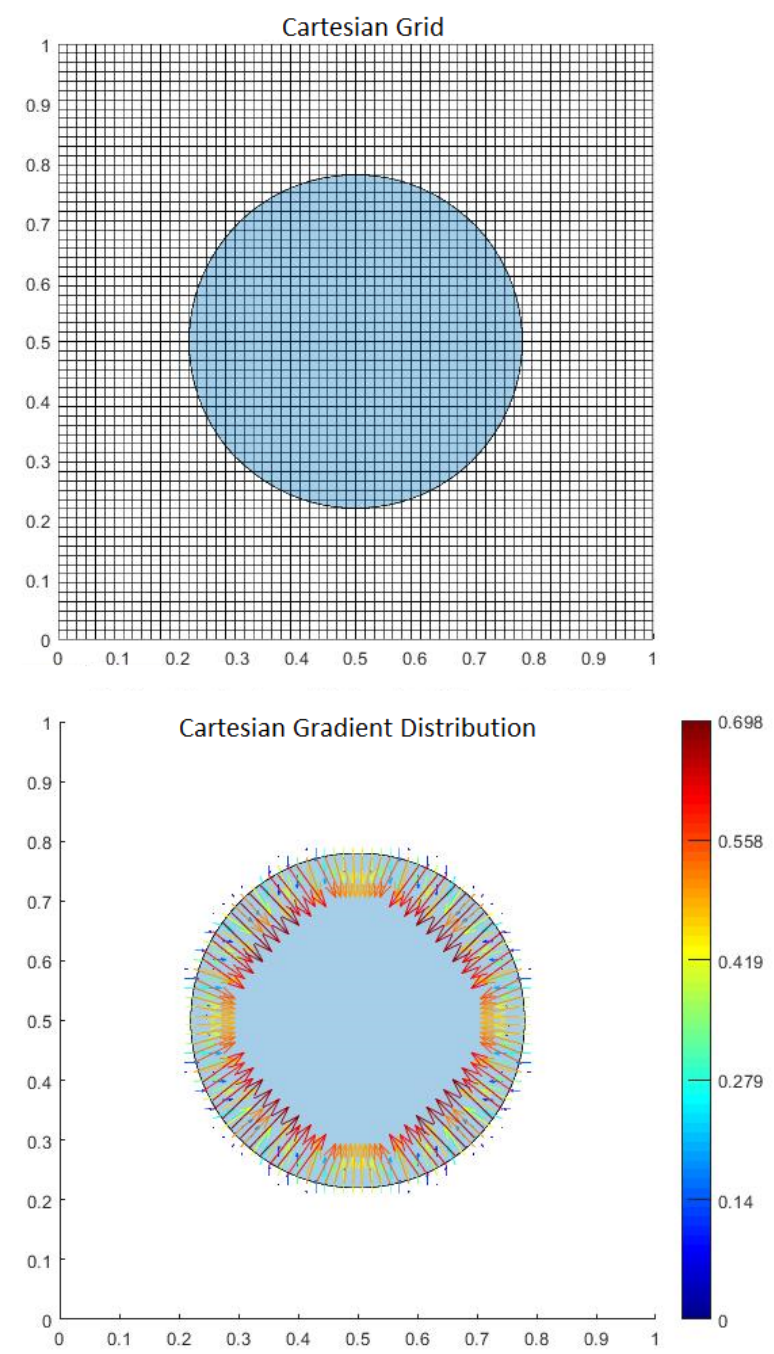

(a)
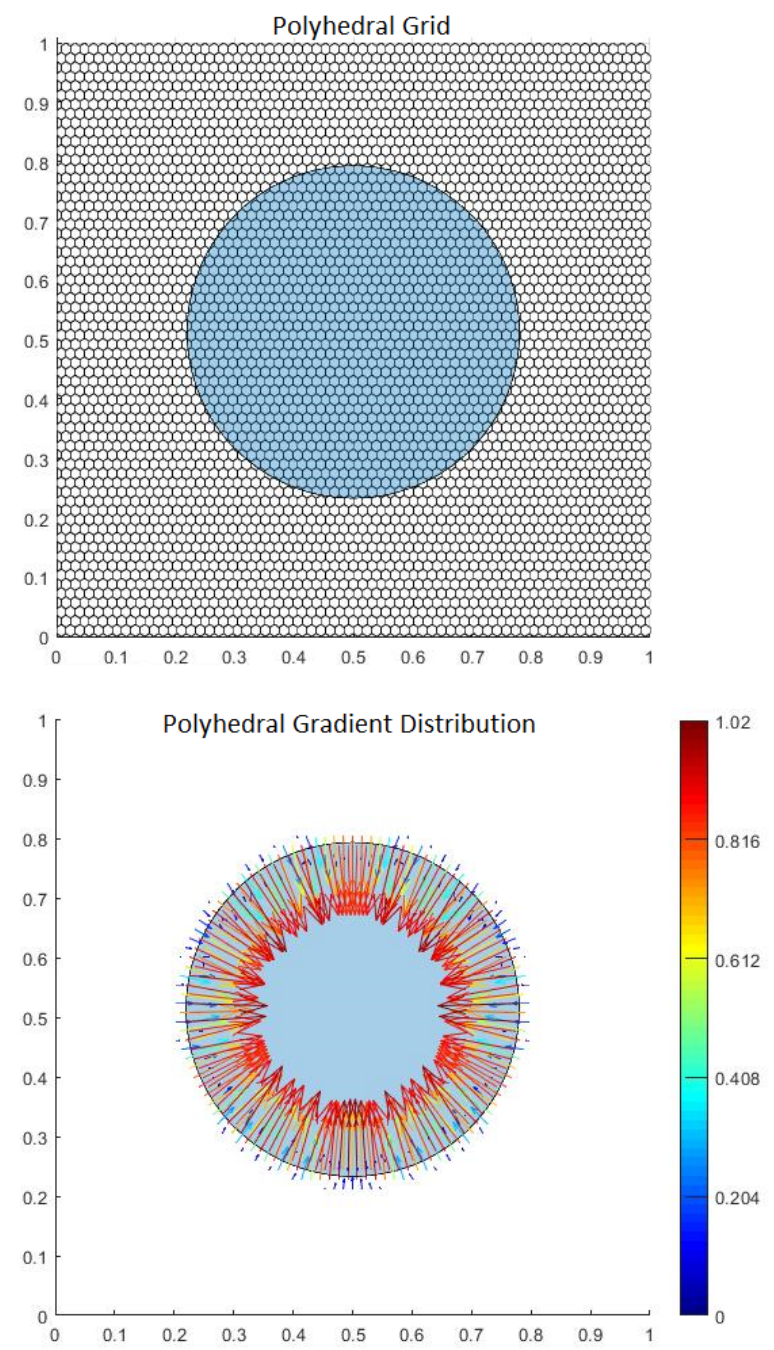

(b)

Figure 2. 2D represantation of gradient calculation on interface. (a) Cell centered gradient is calculated by cartesian cell and 4 neighbor. (b) Cell centered gradient is calculated by polyhedral cell and 6 neighbor.

cells. The magnitude of gradient vector clearly shows elevated values every 90 degree in FIG. 2.

When the distribution of gradient values normalized by the maximum magnitude is analysed as a function of polar angle $\theta$ (FIG. 3a), it is seen on the left side of the figure that there is a difference of up to $25 \%$ between the largest and smallest gradient values calculated on the interface for the cartesian grid. When the distribution of gradient values calculated by polyhedral gradient distribution is examined (FIG. 3b), it is observed that the difference between the largest and smallest gradient values calculated on the interface falls to an average of $8-12 \%$. When the gradient distribution is examined, it is observed that the gradient values peak at every 90 degrees on the 360-degree interface for cartesian grid distribution, and in polyhedral grid, these values are repeated on average at every 60 degrees and with relatively low amplitudes. In the same figure, the grid resolution is also varied and, surprisingly no reduction in the amplitude of oscillations is observed. This simple analysis clearly shows that the grid type and the number of neighboring cells used in gradient calculations are more important than the mesh resolution when calculating the gradient. Moreover, the direction of error accumulation is dependent on the number of the neighboring cells. In other words, in gradient calculations, the polyhedral grid structure would provide smoother distribution of volume fraction gradient compared to the cartesian grid structure. 

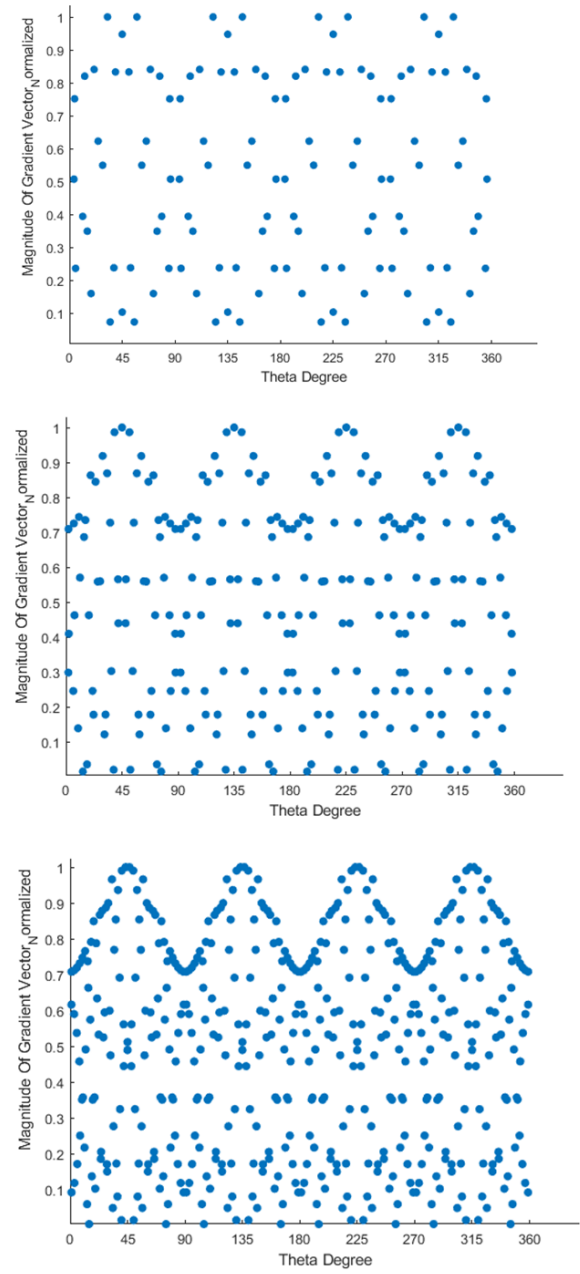

(a)
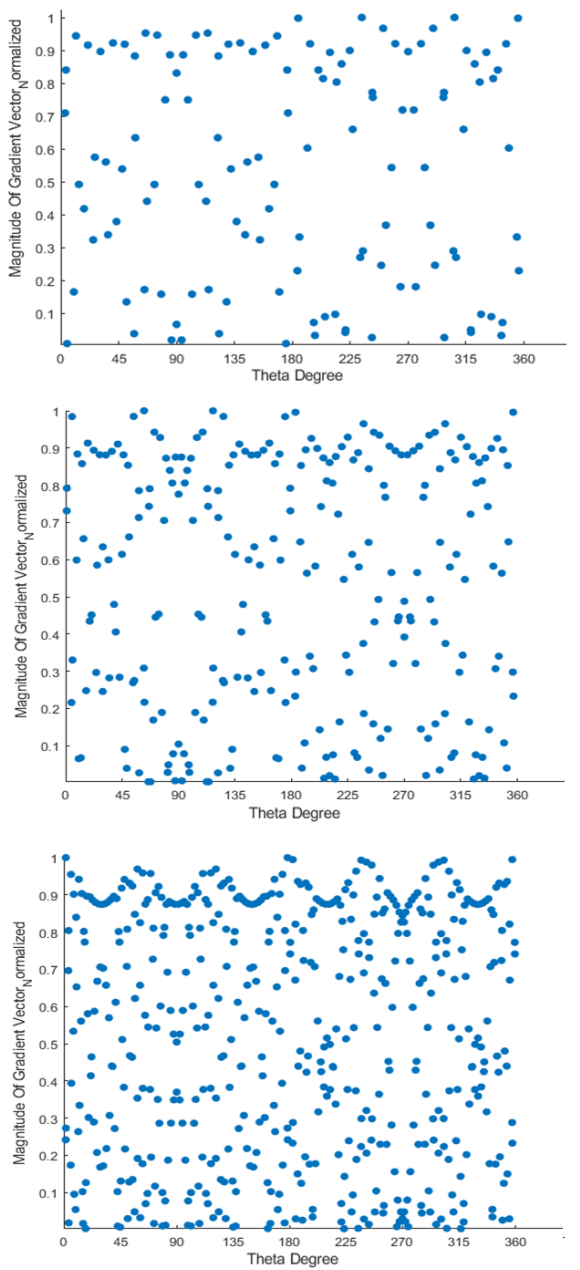

(b)

Figure 3. The 3 comparable analyzes that normalized magnitude of gradient value ordered from top to bottom were calculated with 24x24, 48x48, 96x96 grid resolutions. (a) Cartesian grid results. (b) Polyhedral grid results.

\section{Results and discussion}

In this section, experimental results and simulation results were compared in specific time sequences. Validation of the developed OpenFOAM solver, which utilizes polyhedral mesh stucture, is conducted. The working fluid is water at standard conditions for all the cases. As can be seen, there are two surfaces with high contact angle hysterisis and one surface (Case 1) with low hysterisis. Case 1 is hydrophobic both in advancing and receeding phase, whereas impact to Parafin surface (Cases 2 ) is hydrophobic while advancing and hydrophilic during receeding phase. The ratio of the droplet inertia-surface tension relationship is characterized by non-dimentional $W e_{n}$ we $W e_{t}$ numbers for normal and tangential forces. Impact conditions of Case 1 and Case 2 are given in Table 1.

Stationary smooth surface simulation, surface coating material and their responses at the contact angles specified in the results are shown with physical image and non-dimensional spread factor. Non-dimensional spread factor values are used to compare the drop diameter in the experiments and simulations at the same time sequences. Experiment and simulation spread factor comparisons of static and moving wall conditions can be given in Fig. 5 and Fig 7 respectively. Due to the droplet spreads at the same size in the tangential and radial directions on the static surfaces, it is sufficient to make a comparison of the radial spread factor on the static wall conditions. In the moving wall conditions, both tangential and radial spread factor should be compared. 
Non-dimensional time is defined as:

$$
t^{*}=\frac{v \cdot t}{D}
$$

where $\mathrm{t}$ is time, $\mathrm{v}$ is initial velocity, $\mathrm{D}$ is initial drop diameter.

Table 1. Impact conditions for the validation cases

\begin{tabular}{c|ccccccc}
\hline & Surf. Material & $\mathrm{We}_{n}$ & $\mathrm{We}_{t}$ & Drop. Size & $\theta_{\text {adv }}$ & $\theta_{\text {rec }}$ & $\theta_{\text {eq }}$ \\
\hline Case 1 & Wax & 52 & 0 & $2.75(\mathrm{~mm})$ & $105^{\circ}$ & $95^{\circ}$ & $100^{\circ}$ \\
Case 2 & Paraffin & 42.3 & 14.49 & $0.75(\mathrm{~mm})$ & $113^{\circ}$ & $63^{\circ}$ & $104^{\circ}$
\end{tabular}
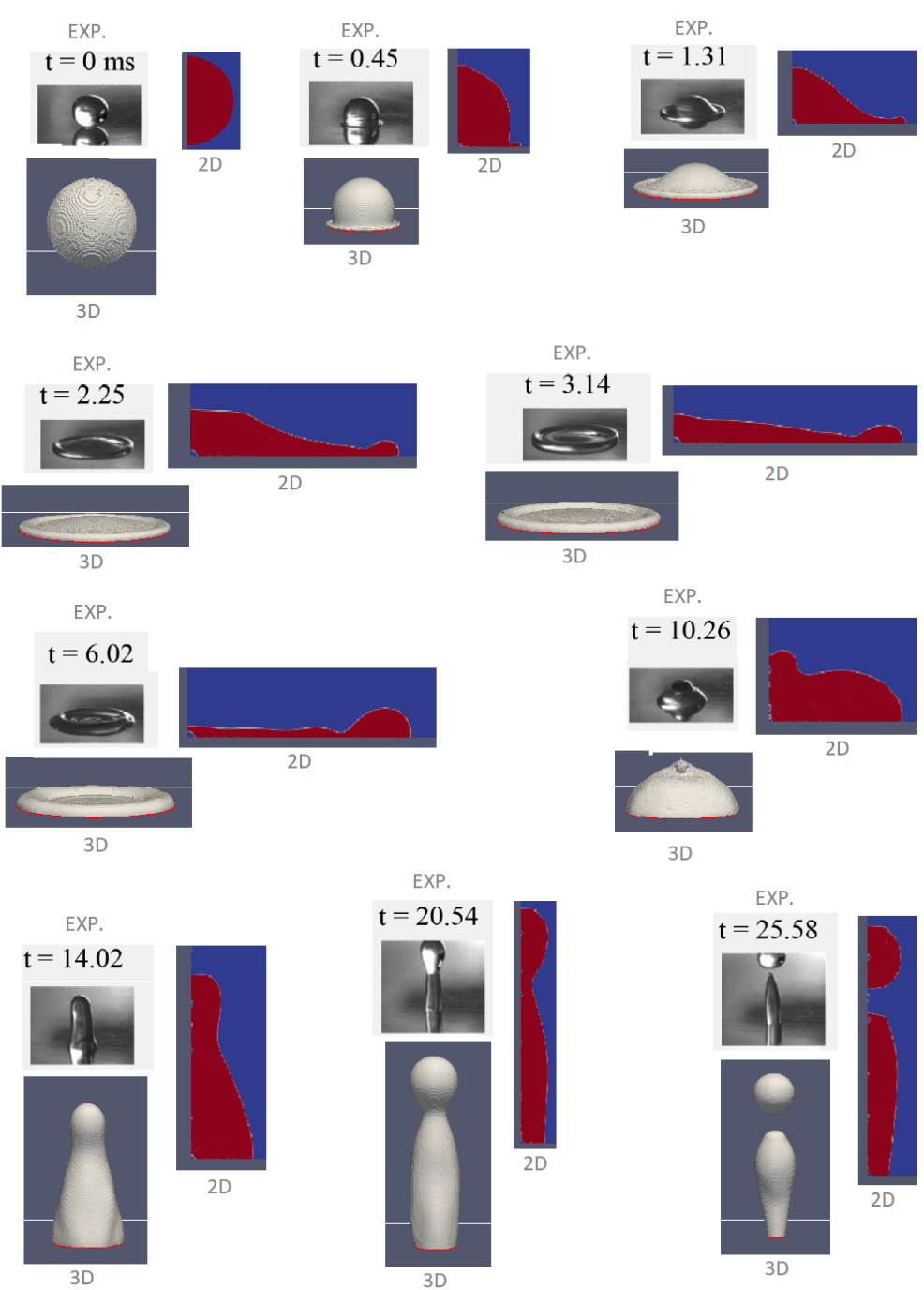

Figure 4. Time evolution of water drop impact on stationary wax surface with partial rebound outcome. Simulated images are extracted from 3D and 2D simulation results (Case 1). Time slots are shown in milliseconds.

Experimental images are taken from Experiments in Fluids, Time evolution of liquid drop impact onto solid, dry surfaces, 33, 2002, pp 112-124, R. Rioboo, M. Marengo and C. Tropea, with permission will be taken of Springer. 


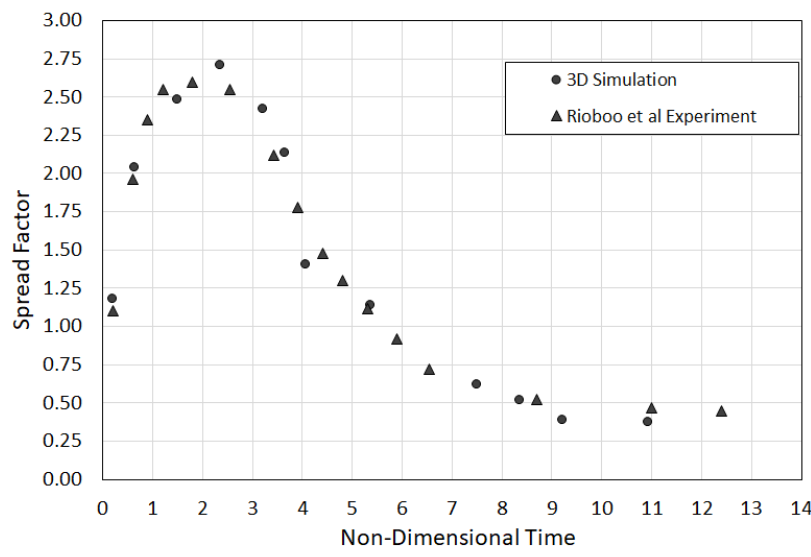

Figure 5. Non-dimensional spread factor versus non-dimensional time showing the evolution of the spread factor for reference partial rebound case (Case 1).
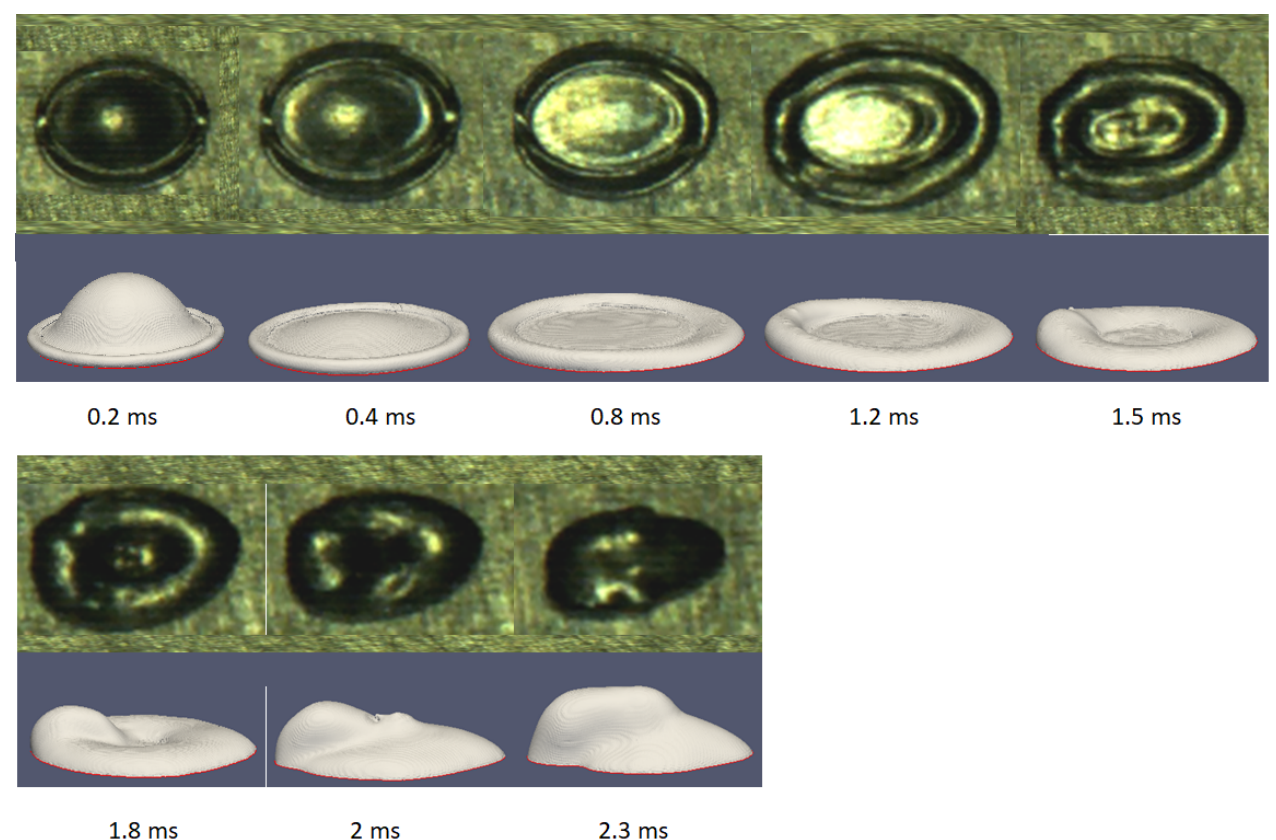

Figure 6. Time evolution of water drop impact on moving wax surface with deposition outcome. Simulated images are extracted from 3D simulation results. Time slots are shown in milliseconds. Experimental images are taken from Kayansalcik [15]
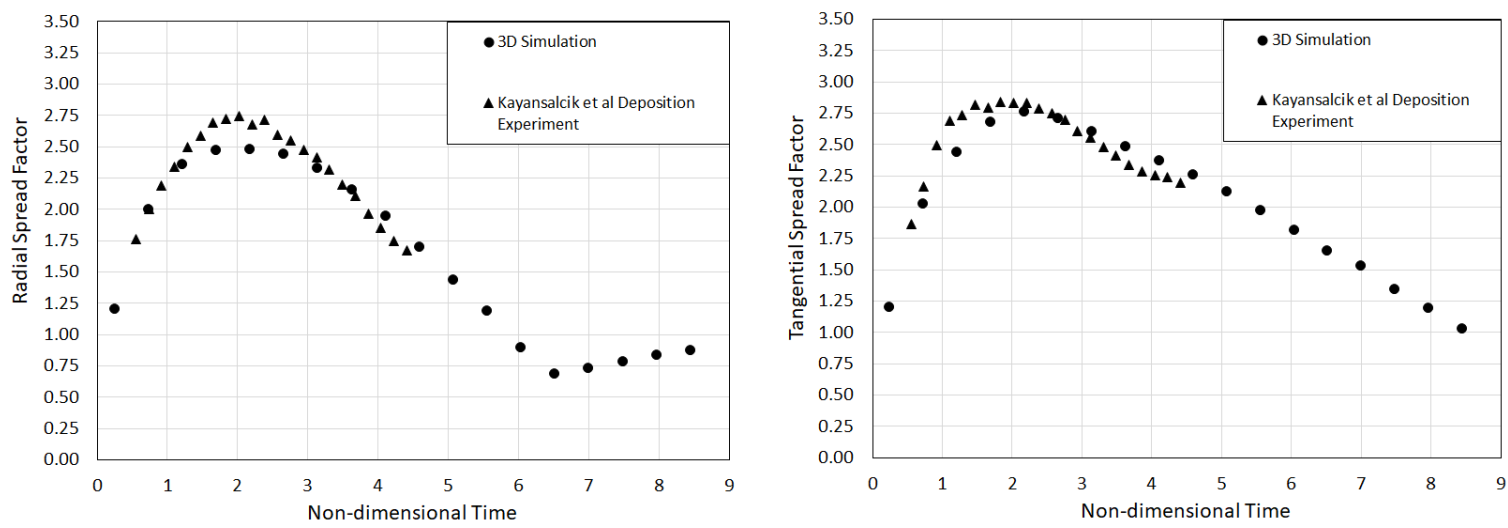

Figure 7. Non-dimensional Radial and Tangential spread factors versus non-dimensional time showing the evolution of the spread factor for reference deposition case (Case 2). 


\section{Conclusions}

In this study, parasitic currents and their causes, which we encounter especially in drop simulations and affect the behavior of the droplet, were investigated. It has been observed that parasitic currents are associated with the grid structure in the spreading plane. With this noticed correlation, it has been seen that the grid resolution has no effect on parasitic currents. The generation of parasitic currents is caused by computational errors of volume fraction gradients at the droplet interface. When the parasitic current and interface deformation were examined, it was observed that the errors accumulate in the spreading plane. Increasing the number of neighbors for each cell in this plane contributed to the more smooth distribution of gradients. Thus, the use of polyhedral cell structure instead of cartesian cell structure contributed to the smooth distribution of interface gradient values. By using polygonal cell, the interface gradients are distributed at lower amplitudes and higher frequencies. Due to the use of polyhendral grid structure in 3D simulations, interface deformations are largely eliminated. Experimental static and moving wall test cases results obtained good results due to parasitic current improvements.

\section{Acknowledgements}

The author would like to gratefully acknowledge Scientific and Technological Research Council of Turkey (TUBITAK) for providing financial support to this research with the 114M423 project.

\section{References}

[1] Chen, R. H., and Wang, H. W., 2005, Experiments in Fluids, 39, pp. 754-760.

[2] Rioboo, R., Marengo, M., and Tropea, C. (2002). Experiments in Fluids, 33(October 2014), 112-124.

[3] Vontas, K., Andredaki, M., Georgoulas, A., Nikas, K. S., and Marengo, M., 2017, Proc. ILASS-Europe 2017. 28th Conf. Liq. At. Spray Syst., (September).

[4] Wildeman, S., Visser, C. W., Sun, C., and Lohse, D., 2016, Journal of Fluid Mechanics., 805 , pp. $636-655$.

[5] Jian, Z., Josserand, C., Popinet, S., Ray, P., and Zaleski, S., 2018, Journal of Fluid Mechanics, 835, pp. 1065-1086.

[6] Harvie, D. J. E., Davidson, M. R., and Rudman, M., 2006,Appl. Math. Model., 30, pp. 1056-1066.

[7] Brackbill, J. U., Kothe, D. B., and Ridge, O., 1992,Journal of Computational Physics, 9991(November 2017).

[8] Popinet, S., 2003,Journal of Computational Physics, 190, pp. 572-600.

[9] Popinet, S., 2015, Journal of Computational Physics, 302, pp. 336-358.

[10] Afkhami, S., and Bussmann, M., 2008, Int. J. Numer. METHODS FLUIDS, 57, pp. 453-472.

[11] Boger, M., Schlottke, J., and Weigand, B., 2010, Reduction of Parasitic Currents in the DNS VOF Code FS3D.

[12] Eisenschmidt, K., Ertl, M., Gomaa, H., Kieffer-roth, C., Meister, C., Rauschenberger, P., Reitzle, M., Schlottke, K., and Weigand, B., 2016,Applied Mathematical Computation, 272, pp. 508-517.

[13] Weller, H. G., Tabor, G., Jasak, H., and Fureby, C., 1999, Journal of Computational Physics, 620.

[14] Berger, M. J., and Colella, P., 1989, Journal of Computational Physics, 82(1), pp. 64-84.

[15] Kayansalcik, G., 2018,Master Thesis, Özyeğin University.

[16] Heidarifatasmi, H., Ertunç, Ö. 2017. Droplet 2017. UCLA, Los Angeles.

[17] Extrand, C. W., Kumagai, Y. 1995, Journal of Colloid and Interface Science ,170(2), 515 521. 